\title{
A User-Centered Approach on Combining Realism and Interactivity in Virtual Environments
}

\author{
Maria Roussou $^{1}$, George Drettakis ${ }^{2}$, Nicolas Tsingos ${ }^{2}$, Alex Reche ${ }^{2,3}$, Emmanuel Gallo $^{2}$ \\ ${ }^{1}$ University College London, \\ ${ }^{2}$ REVES/INRIA \\ ${ }^{3} \mathrm{CSTB}$, France \\ Dept. of Comp. Science, UK \\ Sophia-Antipolis, France \\ http://www.cstb.fr \\ m.roussou@cs.ucl.ac.uk \\ http://www.inria.fr/reves
}

\begin{abstract}
In this paper we describe a project that adopts a usercentered approach in the design of virtual environments (VEs) with enhanced realism and interactivity, guided by real-world applications in the areas of urban planning/architecture and cultural heritage education. In what concerns realism, we introduce an image-based $3 D$ capture process, where realistic models are created from photographs and subsequently displayed in a VR system using a high-quality, view-dependent algorithm. The VE is further enhanced using advanced vegetation and shadow display algorithms as well as 3D sound. A high degree of interactivity is added, allowing users to build and manipulate elements of the VEs according to their needs, as specified through a user task analysis and scenario-based approach which is currently being evaluated. This work is developed as part of the EU-funded research project CREATE.
\end{abstract}

\section{Introduction}

Despite the promise of virtual environments (VEs), their actual use by end-users in areas other than those that are directed to VR experts has typically been limited to date. Our approach combines realism (both visual and auditory) and interactivity. We believe that these two properties, combined with a user-centered design approach, can improve the development of compelling, usable, and useful virtual environments. For this work we have chosen two application domains that relate to learning and working in VR, an archaeological reconstruction in southern Greece and an urban development project, the construction of a tramway through the city of Nice, France. Our choice has been motivated by the fact that both tasks are real-world projects that are currently in progress and in need of high-level tools and presentation means that will facilitate the work or help in better dissemination of their cause.

\section{Creating and Enhancing Realistic VEs}

To create a photorealistic VE, we introduce a novel workflow for capture, enhancement and realistic display (including sound), based on photos of real environments. In computer graphics, a number of Image-Based Modelling and Rendering techniques have been developed (e.g., [1]). Despite recent advances these techniques usually require overwhelming storage and special purpose display methods. We have chosen to use view-dependent texture mapping, with a small number of input images. The data capture workflow starts by choosing a number of viewpoints around the selected activity points. Panoramic images are then created by photographing all around each viewpoint and "stitching" the images together. These panoramas are rendered as cubemaps and loaded into an image-modelling program, such as REALVIZ ImageModeler ${ }^{T M}$. The cameras of images/faces of the cube are calibrated, and a 3D model of the scene is then constructed. This model is the same for all the panoramas used. Textures are then extracted by projecting the image back into object space, and edited for each view. The result of this process is a single $3 \mathrm{D}$ model, and one set of textures per panorama (viewpoint). This allows view-dependent display of the models, resulting in significantly higher quality renderings with low-polygon count models. As an alternative we use a layered image approach [4] from the same data (images and 3D model). The resulting environment can be edited using standard 3D modelling tools, and virtual elements can be added as needed.

A significant factor for realism is the use of viewdependent display: in the first approach we choose the appropriate viewpoint and display the closest texture corresponding to the current observer position, blending in between when necessary. The second approach uses projective textures using layered images [4], avoiding the need to subdivide the geometry according to each viewpoint and reducing texture memory requirements. Realism is further enhanced by correct lighting using integrated illumination from a sky and sunlight model to achieve consistency. We have also added interactive shadow display, by using perspective shadow maps[5], and vegetation, by integrating a mixed point and line-based rendering approach [2]. We have also included a spatialized 3D sound system which dynamically builds clusters of sound sources [6]. This scales well to large numbers of sources (for crowds, traffic etc.). 

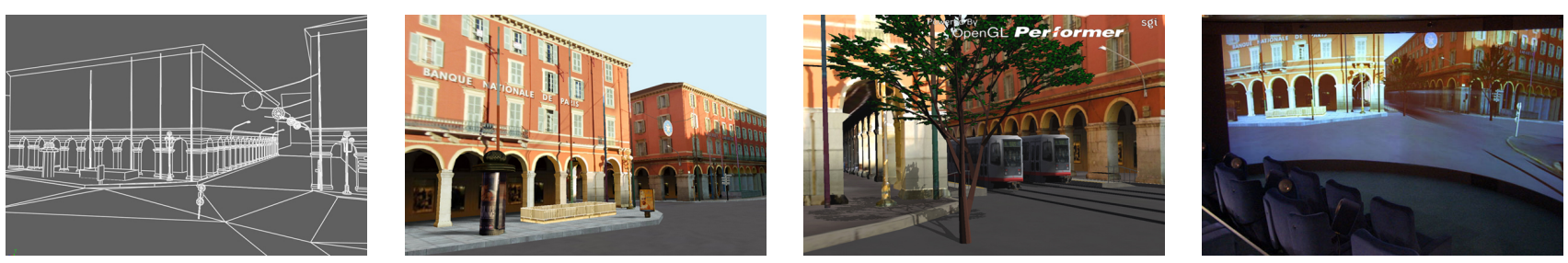

Figure 1. (left to right): wireframe of the 3D model reconstructed from a series of photos; the textured version of the model; the VE after the addition of virtual elements, shadows and lighting; the VE of the urban planning application as displayed on an immersive curved-screen display.

\section{Adding Interactivity}

In addition to the development of visually rich VEs, our user-centered approach presupposes the development of usable applications that include a number of interactive capabilities designed to meet real-world user requirements. The design of interactivity for each case has resulted in a set of representative user task scenarios that are currently being evaluated and refined.

In the case of the archaeological reconstruction scenario, the central temple of ancient Messene in Greece provides a focused activity space in which the user engages in the reconstruction of the temple. Such an activity involves the manipulation of all available architectural members (selecting, resizing, positioning) to virtually "build", examine, and compare, parts of the temple as these are found (and accurately captured) in the environment. The goal is to offer the user the possibility to try varied reconstruction hypotheses and actively explore various levels of reconstruction for different historical periods.

Similar activity has been designed for the urban planning scenario where the addition of interactive features allow users to dynamically investigate alternatives by directly manipulating environmental elements (placement/height of a statue, platforms, vegetation, etc.). The architects of the tramway project in Nice will use the VE's interactive features to make judgments, examine alternative development scenarios, and experiment with different solutions during their virtual prototyping process.

\section{User-Centered Design and Evaluation}

The case studies for this project have been designed with a user-centered approach. Our evaluation methodology is largely based on a structured framework for the design and evaluation of user activity in VEs that includes user task analysis, heuristic and usability guidelines, formative, and summative evaluation [3]. We have extended this framework to take into account the specific application domains of education and design, and the different types of users that our work targets, including children. For both domains, experts (architects, archaeologists, urban planners) and novices (students, museum visitors, city administration, the general public) were selected and interviewed. The results of this needs analysis process have informed the design and development of our prototypes and formative evaluation studies. Exploratory studies were conducted, for the education case, with young students between the age of 7 and 12 . The tasks included simple building activities that involved the selection, placement, and manipulation of virtual objects. Technical and usability evaluation of the prototype environment was also performed. Similar studies are under way with the architects of the urban planning project.

The pilot studies carried out thus far have been very useful in providing directions for the design of the next studies, while user involvement and response has been essential in this process. The addition of haptic interfaces and virtual people are considered important future directions. The user studies will continue, both on a formative and on a summative level, so as to ensure that the resulting application environments meet their users' needs and provide virtual reality tools that address real-world situations.

\section{Acknowledgments}

CREATE is a 3-year project funded by the 5th Framework IST Programme of the European Union. The authors wish to thank all the individuals from the partner institutions that have contributed to the development of the project. http://www.cs.ucl.ac.uk/create/.

\section{References}

[1] P. Debevec, C. Taylor, and J. Malik. Modeling and rendering architecture from photographs: A hybrid geometry- and image-based approach. In Proc. SIGGRAPH 96, 1996.

[2] O. Deussen, C. Colditz, M. Stamminger, and G. Drettakis. Interactive visualization of complex plant ecosystems. In Proc. of IEEE Visualization 02. IEEE, October 2002.

[3] J. L. Gabbard, D. Hix, and J. E. Swan II. User-centered design and evaluation of virtual environments. IEEE Computer Graphics and Applications, 19:51-59, Nov/Dec 1999.

[4] A. Reche and G. Drettakis. View dependent layered projective texture maps. In Proc. of Pacific Graphics 2003, Oct. 2003.

[5] M. Stamminger and G. Drettakis. Perspective shadow maps. In Proc. of ACM SIGGRAPH 2002, July 2002.

[6] N. Tsingos, E. Gallo, and G. Drettakis. Perceptual audio rendering of complex virtual environments. Technical Report RR-4734, INRIA, Feb 2003. 